\title{
Proximity-dependent biotinylation screening identifies NbHYPK as a novel interacting partner of ATG8 in plants
}

\author{
Mercy W. Macharia', Wilfred Y. Z. Tan ${ }^{1}$, Prem P. Das ${ }^{1}$, Naweed I. Naqvi ${ }^{1,2}$ and Sek-Man Wong ${ }^{1,2,3^{*}}$ (D)
}

\begin{abstract}
Background: Autophagy is a conserved, highly-regulated catabolic process that plays important roles in growth, development and innate immunity in plants. In this study, we compared the rate of autophagy induction in Nicotiana benthamiana plants infected with Tobacco mosaic virus or the TMV 24A + UPD mutant variant, which replicates at a faster rate and induces more severe symptoms. Using a BirA* tag and proximity-dependent biotin identification (BiolD) analysis, we identified host proteins that interact with the core autophagy protein, ATG8 in TMV 24A + UPD infected plants. By combining the use of a fast replicating TMV mutant and an in vivo protein-protein screening technique, we were able to gain functional insight into the role of autophagy in a compatible virus-host interaction.

Results: Our study revealed an increased autophagic flux induced by TMV 24A + UPD, as compared to TMV in $N$. benthamiana. Analysis of the functional proteome associated with ATG8 revealed a total of 67 proteins, 16 of which are known to interact with ATG8 or its orthologs in mammalian and yeast systems. The interacting proteins were categorized into four functional groups: immune system process, response to ROS, sulphur amino acid metabolism and calcium signalling. Due to the presence of an ubiquitin-associated (UBA) domain, which is demonstrated to interact with ATG8, the Huntingtin-interacting protein K-like (HYPK) was selected for validation of the physical interaction and function. We used yeast two hybrid $(\mathrm{Y} 2 \mathrm{H})$, bimolecular fluorescence complementation (BiFC) and subcellular localization to validate the ATG8-HYPK interaction. Subsequent down-regulation of ATG8 by virus-induced gene silencing (VIGS) showed enhanced TMV symptoms, suggesting a protective role for autophagy during TMV 24A + UPD infection.

Conclusion: This study presents the use of BiolD as a suitable method for screening ATG8 interacting proteins in planta. We have identified many putative binding partners of ATG8 during TMV 24A + UPD infection in N. benthamiana plants. In addition, we have verified that NbHYPK is an interacting partner of ATG8. We infer that autophagy plays a protective role in TMV 24A + UPD infected plants.
\end{abstract}

Keywords: BioID, Autophagy, ATG8, TMV, TMV 24A + UPD, Protein-protein interaction, HYPK

\section{Background}

Induction of autophagic vesicles has been reported with infection of several viruses in both plant and animal cells [1-4]. Unless impaired, autophagy pathways endeavour to actively eliminate intracellular invaders to promote cell survival [2]. Studies have revealed an important role of autophagy in plant innate immunity, programme cell death, plant resistance and viral RNA silencing [4-6]. Recent

\footnotetext{
* Correspondence: dbswsm@nus.edu.sg

'Department of Biological Sciences, National University of Singapore, Singapore 119543, Singapore

${ }^{2}$ Temasek Life Sciences Laboratory, Singapore 117604, Singapore

Full list of author information is available at the end of the article
}

studies have continued to elucidate roles of autophagy, its basic mechanism and interaction with other host components during virus infection in plants. ATG8 directly interacts and facilitates elimination of Cotton leaf curl Multan virus (CLCuMuV) virulence factor [5] and Cauliflower mosaic virus (CaMV) capsid protein P4 [6]. Calmodulinlike protein $\mathrm{NbCaM}$, interacts with $N$. benthamiana Suppressor of Gene Silencing 3 (NbSGS3) and promotes autophagic degradation during geminivirus infection [7]. During Turnip mosaic virus (TuMV) infection, selective autophagy cargo receptor NBR1 targets the RNA silencing suppressor HCpro, thus suppressing viral accumulation [8]. ATG6 interacts with

(c) The Author(s). 2019 Open Access This article is distributed under the terms of the Creative Commons Attribution 4.0 International License (http://creativecommons.org/licenses/by/4.0/), which permits unrestricted use, distribution, and 
TuMV RNA-dependent RNA polymerase (RdRp) and restricts its infection [9].

RNA viruses exploit cellular machinery into virus assembly complexes through various compositions of protein-RNA, protein-lipid and protein-protein components [10]. Their interaction with plant autophagy is largely unexploited. Therefore, there is a need to identify major host proteins that are involved in autophagy during compatible RNA virus-host interaction. Autophagy induces the formation of autophagosomes which are double-membrane vesicles that engulf cargoes such as proteins, damaged organelles and pathogens and transport them to the vacuoles for degradation [11]. ATG8 is a core protein associated with maturation of the autophagosome [12]. Therefore, screening for ATG8 interacting proteins is crucial in understanding the basic mechanism of autophagosome formation and processing during viral infection.

Tobacco mosaic virus (TMV) is a positive sense RNA virus that is compatible with certain genotypes of tobacco plants. It has an upstream pseudo-knot domain (UPD) at the 3 ' end that plays a crucial role during virus translation and expression [13]. TMV 24A + UPD, a mutant engineered by introducing an internal poly $(\mathrm{A})$ tract $(24$ nucleotides) upstream of the TMV UPD induced earlier and more severe necrotic symptoms [14]. Using this mutant, we investigated autophagy induction and flux in infected $N$. benthamiana plants. We screened for host proteins that interact with ATG8 during mutant virus infection in planta. In this report, we showed that TMV 24A + UPD, a faster replicating mutant, enhanced autophagy induction and flux, as compared to TMV. We employed the BioID technique by fusing the biotin ligase BirA* to ATG8. The technique was initially used in mammalian cells [15] and more recently used in rice protoplasts [16] and detached leaf tissues [17]. The BirA* releases biotinyl-5'-AMP which chemically attaches biotin to proteins that are located within $10 \mathrm{~nm}$ of its proximity. Subsequently, proteins that are close to or interacting with the target protein ATG8 are biotinylated. They can be purified using streptavidin and analysed by tandem mass spectrometry (MS/MS).

A total of 67 proteins were identified, most of which are previously unknown to interact with autophagy proteins in plants. Sixteen of the proteins have been previously shown to directly or indirectly associate with autophagy pathway in animal and/or yeast system. Due to the presence of the UBA domain, which is known to interact with ATG8, the Huntingtin-interacting protein K-like (HYPK) was selected for further validation. Using yeast-two-hybrid $(\mathrm{Y} 2 \mathrm{H})$, biomolecular fluorescence complementation (BiFC) and subcellular co-localization, we showed that HYPK and ATG8 interacted in N. benthamiana plants. Knockdown of NbHYPK did not enhance viral symptoms, in contrast to the observed decrease in symptoms upon autophagy knockdown, suggesting that NbHYPK alone does not influence viral symptoms in test plants. The full length primary sequences of mammalian HYPK and NbHYPK-like proteins share a $57 \%$ similarity of conserved and similar amino acids. Both of the proteins have a conserved UBA domain at the $\mathrm{C}$ terminus. This shared similarity suggests that NbHYPK may play a role similar to the mammalian ortholog. The mammalian HYPK is an intrinsically unstructured chaperone that interacts with Huntingtin (HTT) and it enhances autophagy flux and increases in conversion of ATG8 to the lipidated/active ATG8-PE (LC3I to LC3II) [18].

\section{Results}

TMV 24A + UPD induced autophagosome biogenesis and autophagic flux

TMV 24A + UPD replicated faster and induced more severe symptoms, as compared to TMV [14]. We hypothesized that rapid virus replication could increase autophagy and/or autophagic flux. To determine whether autophagy is triggered upon infection by TMV and TMV 24A + UPD, we detected autophagic flux by confirming the presence of ATG8-II at 2 and 3 dpi (Fig. 1a; Additional file 1: Figure S1). Next, we investigated the ultrastructural changes in infected leaves by transmission electron microscopy (TEM). Membranous vesicles resembling autophagosome-like structures were observed in ultrathin sections of TMV 24A + UPD infected leaves (Fig. 1b). To verify autophagy induction, we infiltrated RFP-ATG8 into virus-infected $N$. benthamiana leaves, and quantified the number of epifluorescent foci (Fig. 1c).

The number of autophagosome-like structures in TMV 24A + UPD infected plants were significantly higher than those in TMV infected plants (Fig. 1d). A significantly higher number of RFP-ATG8 labelled aggregate structures were observed in TMV $24 \mathrm{~A}+\mathrm{UPD}$, as compared to that of TMV (Fig. 1e).

\section{Identification of biotinylated proteins}

After determining that TMV 24A + UPD induced autophagic flux, we sought to identify ATG8 interacting proteins during infection. Two plasmids, PXY01-RFP-HA-BirA* $\left(\right.$ BirA $\left.^{*}\right)$ used as a control and PXY01-RFP-HA-BirA*ATG8 (BirA*-ATG8) used as baits, were constructed (Additional file 1: Figure S2a). Expression of BirA* and BirA*-ATG8 were successful for both constructs (Fig. 2a; Additional file 1: Figure S2b, 2c, 2d, 2e). $N$. benthamiana leaves were agroinfiltrated either with $\mathrm{Bir}^{*}$ or $\operatorname{Bir} \mathrm{A}^{*}$ ATG8 vectors, with agroinfiltration buffer supplemented with $2 \mathrm{mM}$ biotin. Biotinylated proteins were detected by Western blot and purified using Magnetic Dynabeads ${ }^{\mathrm{me}}$ coated with streptavidin (Fig. 2b, c; Additional file 1: Figure S3a, b). The BirA*-ATG8 bound fraction showed multiple biotinylated protein bands (Fig. 2c; Additional file 1: Figure S3c). This suggests that there 

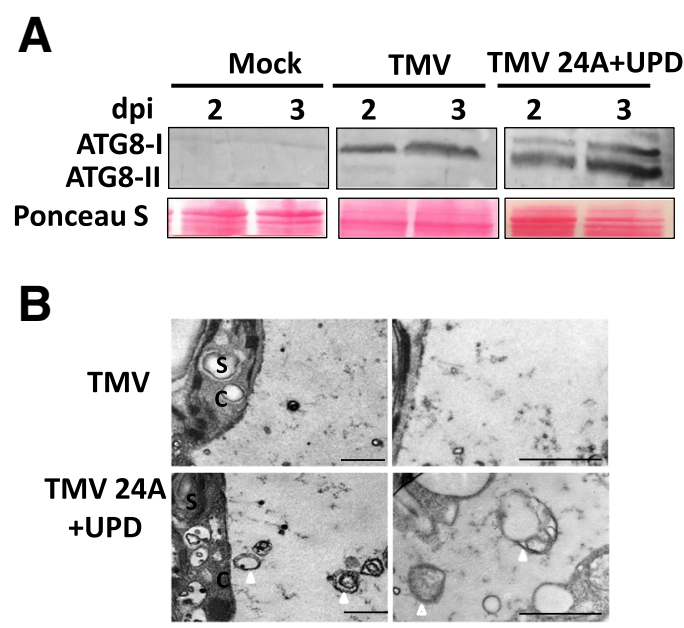

C

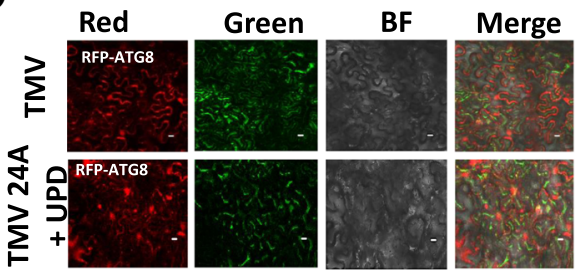

D

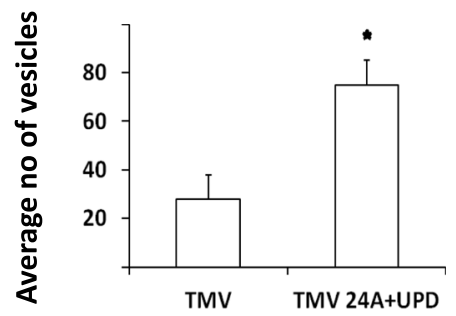

E

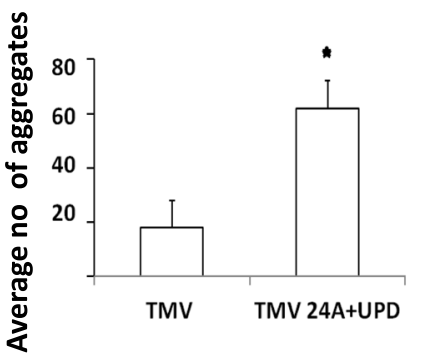

Fig. 1 Autophagy activation in response to TMV and TMV24A + UPD infection in N. benthamiana. a Immunoblot analysis using ATG8 antibody. Molecular weight of the bands detected at $\sim 16 \mathrm{kDa}$. The Ponceau S staining served as a loading control (lower panel, Ponceau S). b Representative electron micrographs showing infected plants at 3 dpi (days post inoculation). Arrows show membranous autophagosome-like structures. Abbreviations; S, starch, C, cytoplasm. c Representative images of RFP-ATG8 labelled autophagosomes at 3 dpi. $\mathbf{d}$ The number of autophagosome-like structures was obtained from $\sim 100 \mathrm{\mu m}^{2}$ section for each experiment. Autophagosome-like structures were significantly enhanced by infection with TMV $24 \mathrm{~A}+$ UPD $\left({ }^{*} p<0.05\right)$. e The mean number of fluorescent puncta/aggregates were obtained from sections with approximately equal number of cells $(\sim 100)$ for each experiment. RFP-ATG8a autophagosomes were significantly enhanced by infection with TMV24A + UPD $\left({ }^{*} p<0.05\right)$. The asterisks indicate significant differences by unpaired sample $t$ test. The scale bar represents $1 \mu \mathrm{m}$

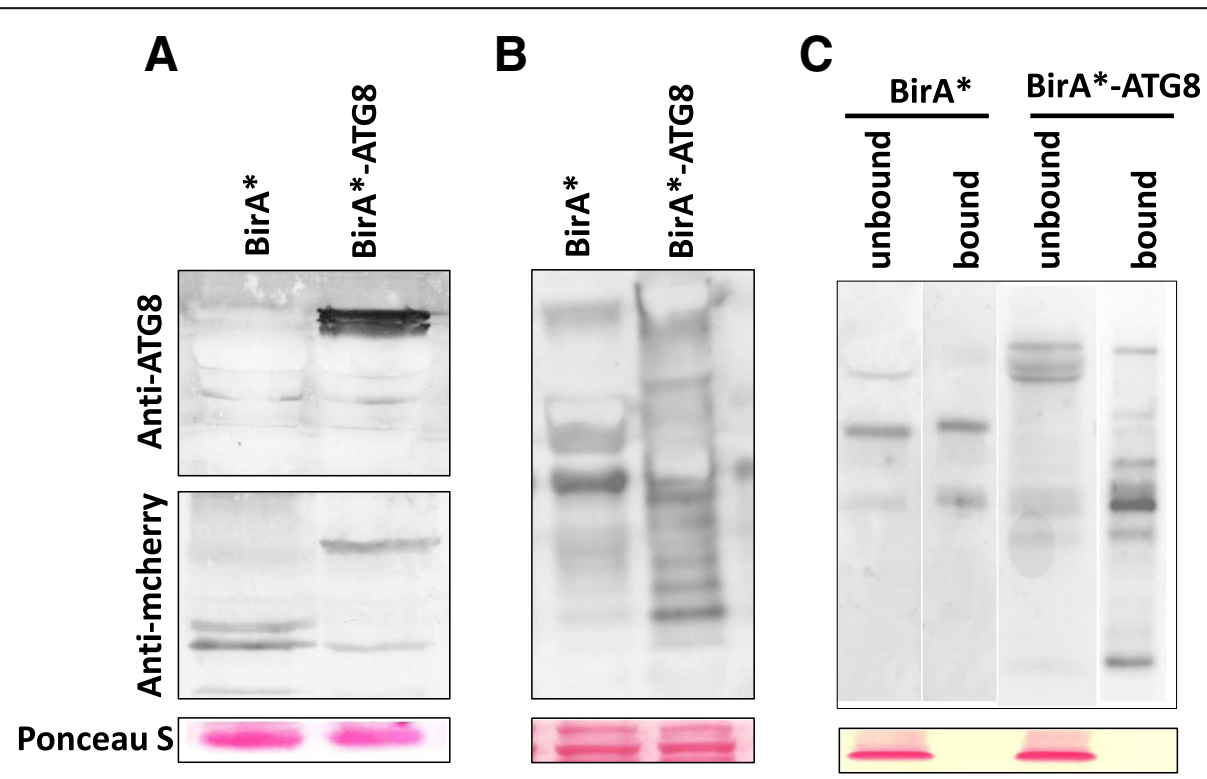

Fig. 2 Immunoblot analysis of BioID fusion proteins and total biotinylated proteins. a Western blots were carried out using anti-ATG8, or anti-mCherry antisera to ascertain fusion protein expression. b Streptavidin-HRP blot of crude protein lysate. c Strep-HRP blot after Dynabeads ${ }^{T M}$ purification. Lower panels show Ponceau S stained blot membranes to confirm equal loading of total proteins 
were more biotinylated proteins in the beads fraction of BirA*-ATG8 samples, as compared to the control.

\section{ATG8 potentially interacts with a network of proteins involved in plant defense}

Subsequent bioinformatics analysis of MS/MS results revealed 5 proteins that were shared between the BirA* and BirA*-ATG8 extracts and 67 proteins were unique to BirA*-ATG8 (Fig. 3a). Gene ontology analysis categorized BirA*-ATG8-identified proteins into 4 functional groups namely: immune system process, response to ROS, sulphur amino acid metabolism and calcium ion signalling (Fig. 3b). Of these proteins, 14 were uncharacterized and 23 were not reported to be involved in autophagy or plant defense (Additional file 2: Table S1). Therefore, they were not selected as candidates for further validation. The rest of the proteins identified were deemed significant, based on published literature (Table 1). These proteins may interact directly or indirectly with ATG8 in N. benthamiana plants. The first 16 proteins have been previously shown to

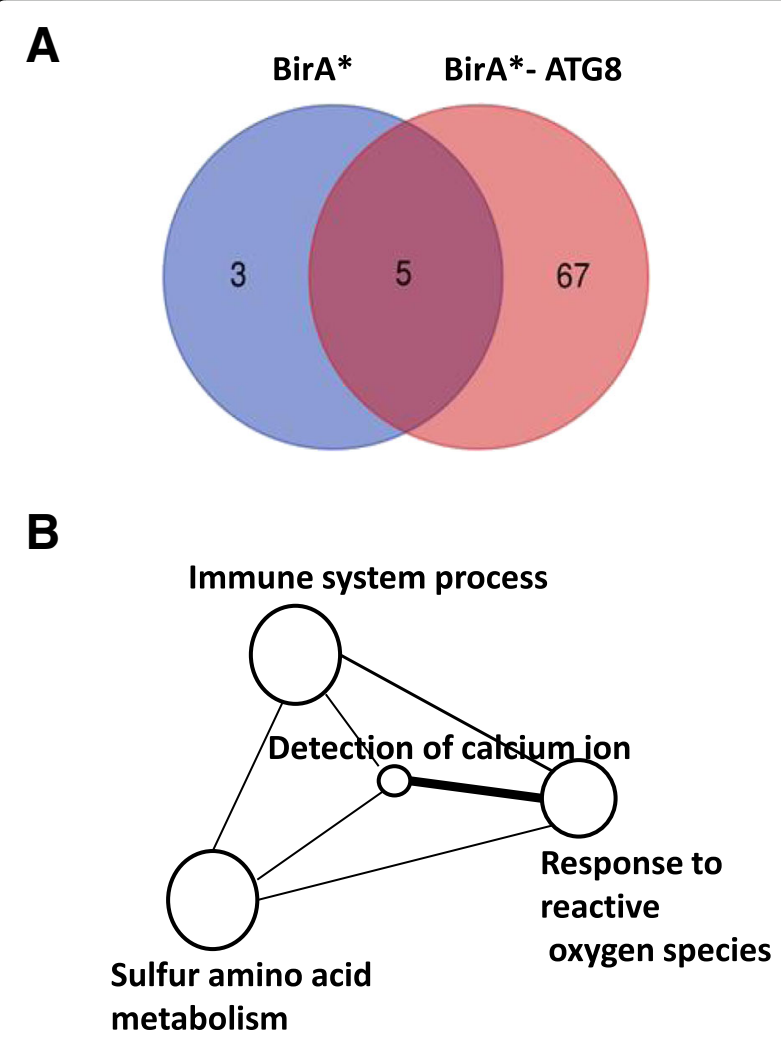

Fig. 3 Statistics of proteins identified and Gene ontology (GO) enrichment analysis of unique BirA*-ATG8 proteins. aVenn diagrams showing unique and shared proteins between BirA* and BirA*-ATG8. b Interactive graphs of proteins that were identified with BirA*-ATG8 during TMV 24A + UPD infection. The sizes of the circles are proportional to the number of proteins associated with the specific term. The interactive network were summarized and plotted following published REVIGO protocol (http://revigo.irb.hr/) interact with the autophagy pathway (Table 1). Proteins 17 to 30 have not been shown to interact with autophagy processes but have biological roles in plant defense and may likely interact with the core autophagy components. We selected the HYPK protein for validation of ATG8 interaction because it contains a UBA domain which has been demonstrated to interact with ATG8.

\section{NbHYPK interacts with ATG8}

NbHYPK was identified from BioID screening and verified by cDNA cloning and sequencing. NbHYPK is a small protein spanning 109 amino acids. It has a UBA domain between amino acids 68-109 and is denoted as UBA NbHYPK. In order to test if the UBA domain is required for its interaction with ATG8, it was deleted from the NbHYPK gene and referred to as NbHYPK $\triangle$ UBA (1-67 aa). We confirmed expression of full length and truncated fusion proteins of HYPK-GFP and RFP-ATG8 by Western blotting (Additional file 1: Figure S4a). We then conducted a co-localization assay After NbHYPK-GFP / NbHYPK $\triangle U B A-G F P /$ UBA_NbHYPK-GFP and mCherryATG8 were co-infiltrated into $N$. benthamiana leaves and transiently expressed, aggregates were observed only in leaves co-expressing NbHYPK-GFP and mCherry-ATG8 (Fig. 4a), but with no aggregates observed in leaves expressing NbHYPK $\triangle \mathrm{UBA}$ or UBA_NbHYPK-GFP and mCherry-ATG8 (Additional file 1: Figure S4b). To further confirm the interaction between NbHYPK and ATG8, we performed a BiFC assay. Constructs with NbHYPK fused to the C-terminus of yellow fluorescent protein (NbHYPK -Yc) and ATG8 fused to the N-terminus of YFP (ATG8Yn) were co-expressed in $N$. benthamiana leaves and resulted in reconstitution of YFP (Fig. 4b). Leaves expressing ATG8-Yn with UBA_NbHYPK -Yc, NbHYPK $\triangle$ UBA -Yc or other empty vector controls failed to generate epifluorescent signals (Additional file 1: Figure S4c).

Additionally, we employed $\mathrm{Y} 2 \mathrm{H}$ to confirm the interaction between NbHYPK and ATG8. ATG8 was fused to the binding domain (BD) and tested for its interaction with NbHYPK fused to the activation domain (AD). Y2H assays validated the physical interaction between NbHYPK and ATG8 (Fig. 4c). We also constructed two other plasmids, NbHYPK $\triangle U B A$ and UBA_NbHYPK, into the AD and tested for their interactions with BD-ATG8. No interaction was evident (Fig. 4c). Taken together, we conclude that the newly identified host protein NbHYPK physically interacts with ATG8 in vitro and in vivo. In addition, we demonstrated that the UBA domain in NbHYPK is essential for such physical interaction with ATG8.

\section{Autophagy protects $N$. benthamiana plants against rapid} cell death induced by TMV 24A + UPD

To test whether autophagy was required for TMV 24A + UPD induced cell death, we silenced ATG5, ATG7 and 
Table 1 BirA*-ATG8 associated proteins identified via BiolD

\begin{tabular}{|c|c|c|}
\hline No. & Accession & Protein name \\
\hline 1. & I7GVS5 & Heat shock protein 70 (Hsp70) $[18,19]$. \\
\hline 2 & A2PYH3 & Alpha chain of nascent polypeptide associated complex (NAC) [20]. \\
\hline 3 & A0A1J6JLR9 & Eukaryotic translation initiation factor $4 \mathrm{~g}$ [21]. \\
\hline 4 & A0A1P8SF07 & TOM1-like protein 2 (Tom1L2) [22] \\
\hline 5 & Q4QXL9 & Autophagy-related Protein 3 (ATG3) [23, 24]. \\
\hline 6 & A0A1U7YMI0 & Glyceraldehyde-3-phosphate dehydrogenase (GAPDH) [24]. \\
\hline 7 & A0A1S3ZA42 & FK506-binding protein 5 (FKBP5)-like isoform X2 [25]. \\
\hline 8 & A0A1U7XHY8 & Subtilisin-like protease (SLP) [26]. \\
\hline 9 & Q8W183 & Carbonic anhydrase [27]. \\
\hline 10 & Q76MF3 & Calmodulin [28]. \\
\hline 11 & A0A1U7YG19 & Serine hydroxymethyltransferase (SHMT) [29]. \\
\hline 12 & A0A1U7VJK4 & Plasminogen activator inhibitor 1 (PAl-1) RNA-binding protein-like [30]. \\
\hline 13 & A8UDS9 & Copper/zinc superoxide dismutase (SOD1) [31]. \\
\hline 14 & A0A1U7XPN3 & Huntingtin-interacting protein K (HYPK) [32] . \\
\hline 15 & A0A1S3XIE4 & Acyl-CoA-binding domain-containing protein 3-like [33]. \\
\hline 16 & A0A1U7XC68 & Clathrin light chain [34]. \\
\hline 17 & A0A1P8SF08 & AvrPto-interacting protein 1 (Api1) \\
\hline 18 & A0A1J6K7H0 & Elongation factor 1-alpha (eEF1A) \\
\hline 19 & A0A1U7XRQ0 & H/ACA ribonucleoprotein (RNP) complex subunit 1-like isoform X2 [35]. \\
\hline 20 & Q95XX4 & Fructose-bisphosphate aldolase [36] \\
\hline 21 & A0A1U7YCY5 & Epidermal growth factor receptor substrate 15 (EPS15)-like 1 \\
\hline 22 & Q6K0Q3 & Chloroplast glutamine synthetase (GS) $[37,38]$. \\
\hline 23 & C9DFB0 & ASR \\
\hline 24 & Q8LKF9 & SGT1-like protein \\
\hline 25 & A0A1U7YZQ4 & probable ADP-ribosylation factor GTPase-activating protein (Arf GAP) AGD6 \\
\hline 26 & A0A1U7YFQ9 & ATP-dependent RNA helicase SUPV3L1, mitochondrial-like \\
\hline 27 & A0A1U7XQL2 & 40 S ribosomal protein S20-2 (RPS20) \\
\hline 28 & A0A1U7WRW2 & 40 S ribosomal protein S6 (RPS6) [39] \\
\hline 29 & A0A1U7VRZ1 & Charged multi-vesicular body protein 5 (CHMP5)-like \\
\hline 30 & A0A1U7VDD8 & Actin cytoskeleton-regulatory complex protein PAN1-like isoform X1 [40]. \\
\hline
\end{tabular}

ATG8, which are core components of autophagy. Such autophagy silenced plants infected with TMV showed increased chlorosis but did not show severe cell death symptoms (Fig. 5a). Cell death triggered by TMV 24A + UPD was markedly increased in ATG5 and ATG7 silenced plants (Fig. 5b). Despite targeting the conserved regions of ATG8 isoforms, ATG8 silencing was less efficient, as compared to ATG5 and ATG7 in both TMV and TMV 24A + UPD infected plants (Additional file 1: Figure S5a, b). Perhaps transient ATG8 silencing was less efficient as ATG8 has 8 isoforms in $N$. benthamiana [41]. The silenced TMV 24A + UPD infected plants showed enhanced cell death symptoms in the newly emerged leaves (Fig. 5b). We infer that autophagy plays a protective role in TMV 24A + UPD infected plants. NbHYPK-silenced plants did show slight but not any obvious differences in viral symptoms, as compared to the normal NbHYPK expressing plants (Fig. 6a). No significant difference was found in coat protein gene expression between NbHYPK-silenced and control plants (Additional file 1: Figure S6a), as quantified by ImageJ [42]).

\section{Discussion}

Several studies have defined autophagy in yeast and mammalian systems [25, 29]. However, autophagy pathways in plants have not been elucidated extensively. In this study, we compared the autophagy induction and flux by TMV and a fast replicating mutant TMV 24A + UPD in $N$. benthamiana plants. An increase of autophagy flux was detected at 2 and 3 dpi (Fig. 1a). Significantly higher number of autophagosome-like structures were detected in plants infected with the faster replicating TMV $24 \mathrm{~A}+$ 
A

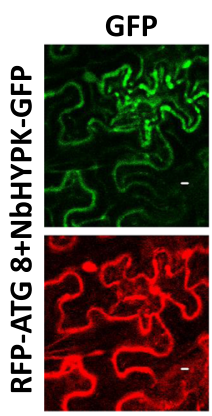

RFP
BF

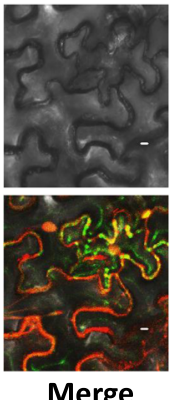

B

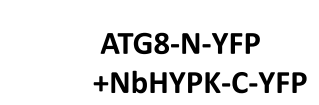

ATG8-N-YFP

+ NbHYPKDUBA-C-YFP

ATG8-N-YFP +UBA_NbHYPK-C-YFP

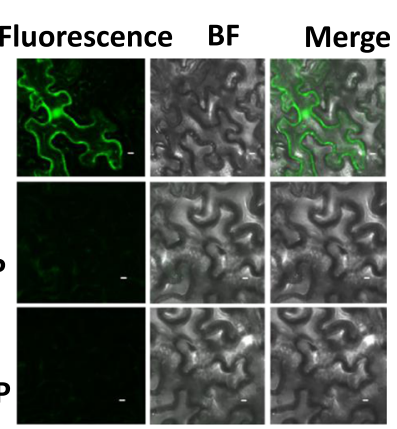

C

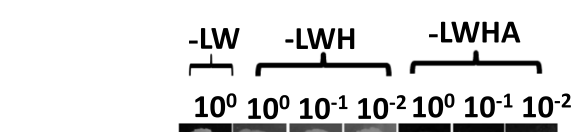

BD+AD

BDATG8+AD

BD+ AD-NbHYPK

BD-ATG8+AD-NbHYPK BD-ATG8+AD-NbHYPK $\triangle$ UBA

BD-ATG8+AD-UBA_NbHYPK

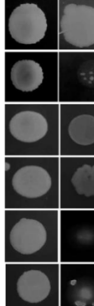

Fig. 4 ATG8 directly interacts with NbHYPK. a GFP labelled NbHYPK, NbHYPKAUBA and UBA-NbHYPK was co-infiltrated with RFP-labelled ATG8 and localized by confocal microscopy. b Bimolecular fluorescence complementation (BiFC) analysis, ATG8-Yn or X-Yn(empty vector) was coexpressed with NbHYPK-Yc, NbHYPKAUBA-Yc and UBA-NbHYPK-Yc or $x-Y c$ (empty vector). Fluorescent signals were visualized after 3 dpi by confocal microscopy. Bar $=1 \mu \mathrm{m}$. c Analysis of interaction between ATG8 and NbHYPK by Yeast two hybrid assay. Plasmids expression full length NbHYPK or the UBA domain or NbHYPK $\triangle$ UBA or empty vector were transformed into AH109 yeast and mated with yeast expressing plasmids containing ATG8 or an empty vector and grown in amino acid drop out YPDA media

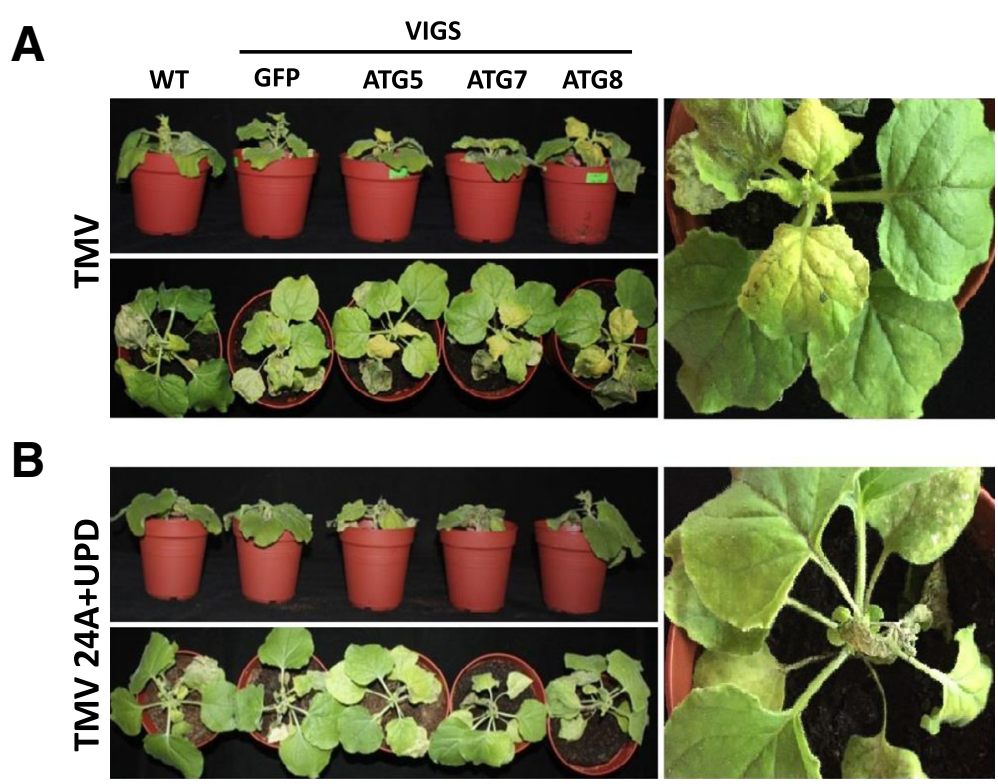

Fig. 5 Silencing important gene(s) essential for autophagy, accelerated TMV 24A + UPD cell death symptoms. aTMV infected plants showing chlorosis in autophagy silenced plants. Inset is an enlarged section of ATG7-silenced plants. b TMV 24A + UPD showing accelerated cell death in autophagy silenced plants. An enlarged ATG7 silenced plant showing accelerated cell death in the emerged leaves 


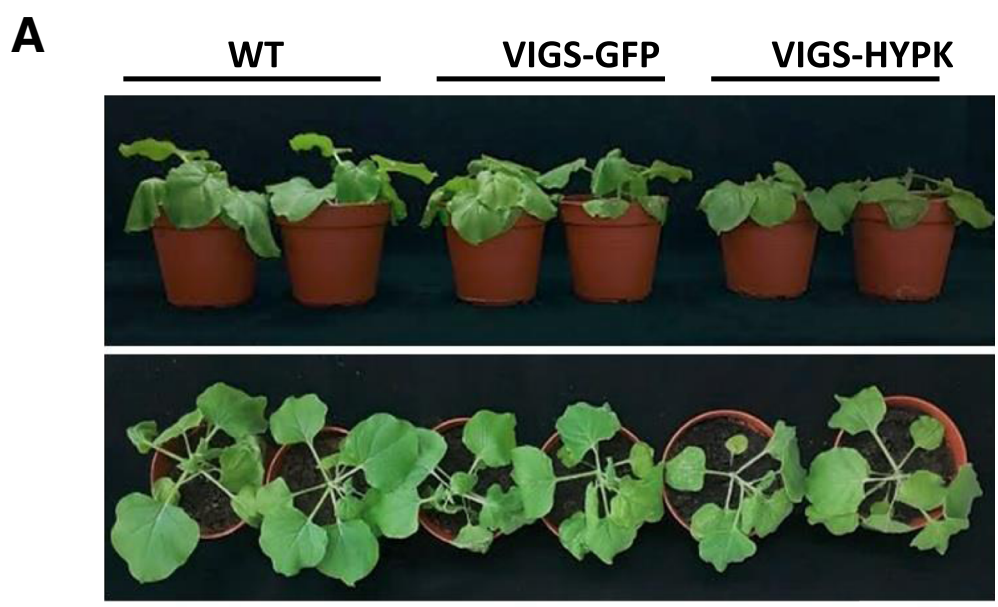

B

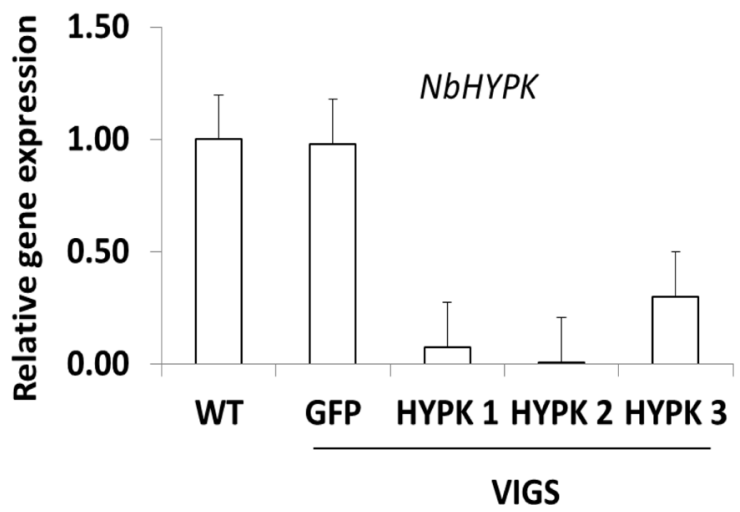

Fig. 6 TMV 24A + UPD symptoms and gene expression analysis in VIGS-NbHYPK plants. a VIGS-silenced plants showed no significant difference in viral symptoms as compared to non-silenced plants. $\mathbf{b}$ Quantitative real-time (qrtPCR) analysis of NbHYPK gene in control and VIGS-NbHYPK plants

UPD variant (Fig. $1 \mathrm{~b}-\mathrm{e})$. This indicates that induction of autophagy is likely accelerated by increased viral load due to a more rapidly replicating TMV virus.

Out of the 67 putative ATG8 interacting proteins identified through BioID screening, 16 proteins were directly associated with autophagy pathways (Table 1). Among these, ATG3 and calmodulin have been shown to interact directly with ATG8 and autophagosomes in N. benthamiana plants [7, 23]. A subtilisin-like protease implicated in programmed cell death and defense signaling in plants, plays a role in animal autophagy [25, 41, 42]. Acyl-CoAbinding domain-containing protein 3-like has been shown to disrupt autophagosome formation and increase ATG8 degradation in plants [33]. Most of the other proteins identified in this group are involved with autophagy in mammalian systems but have not been reported in plants. On the other hand, proteins 17 to 30 (Table 1) are involved in autophagy-associated functions such as pathogen defense, stress signalling, and hypersensitive response (HR) $[4,42,43]$. Proteins 1 to 24 (Additional file 2: Table S1) have biological functions that are not associated with autophagy. Recent studies have revealed autophagy- independent functions of ATG8 in maintaining lipid droplet integrity in yeast and in apicoplast formation in malaria [44-46]. Perhaps ATG8 isoform(s) also play an autophagy-independent role in plants. Some proteins that interact with ATG8, such as ATG4 and ATG7, were not identified through MS/MS. Some autophagy proteins may be difficult to purify as they unstable [47]. In addition, some proteins not interacting with ATG8 were also biotinylated due to their incidental proximity to the BirA*ATG8 fusion protein in cells, an inherent limitation of the BioID technique. Therefore, interaction studies such as $\mathrm{Y} 2 \mathrm{H}, \mathrm{BiFC}$ and co-localization were further considered to confirm the physical interactions.

High throughput protein-protein interaction studies are crucial in narrowing down candidates to study autophagy. ATG8 interacting proteins typically possess an AIM/LIR motif. While this motif is used to select candidates, it does not always represent functionality of the motif [48]. Therefore, additional criteria are employed, such as structural and biological aspects of the proteins. Autophagy receptors Joka2, P62 and NBR1 have UBAlike domain(s) that are crucial in their interaction with 
ATG8 [49]. Analysis of the ATG8 interactor NbHYPK (this study) revealed that the primary sequence does not contain a conventional AIM motif. However, it contained a UBA domain in the C-terminus. Thus, we opted to further validate NbHYPK interaction with ATG8 using the aforementioned analyses.

Our results showed that NbHYPK and NbATG8 interacted physically. The interaction of NbHYPK and ATG8 requires the UBA domain. The UBA domain of NbHYPK has high similarity with another cytosolic chaperone, nascent polypeptide-associated complex proteins (NAC), which is associated with post translational processes of nascent proteins emerging from the ribosomes [50]. We also identified the alpha chain of nascent polypeptide associated complex protein (Table 1). The mammalian Plic-2 protein possesses a UBA domain and it is involved in autophagy [51]. Plic-2 has played a protective role during starvation and requires ATG5 and ATG7 proteins. It directly interacts with ATG8 (LC3) and plays a role in fusion of autophagosomes to lysosomes [51].

Although down-regulation of NbHYPK using VIGS produced no significant difference in viral symptoms and cell death in NbHYPK-silenced plants, silencing NbHYPK may not produce phenotypes but may have an effect on other cellular processes, as evidenced by the mammalian HYPK ortholog. The mammalian huntingtin-interacting protein $\mathrm{K}$ (HYPK) has chaperone-like properties and is involved in cell death, unfolded protein response, cell cycle and cell growth [52]. Overexpressing HYPK increased conversion of the mammalian homologues of ATG8 and ATG8-PE, LC3I to LC3II, respectively [18]. Interestingly, the Huntingtin protein itself (HTT) has been shown to interact with ATG8 and is important for autophagy in Drosophila [32]. We do not rule out the redundancy in chaperone functions in targeting ATG8.

On the other hand, knockdown of ATG5, ATG7 and ATG8 revealed that autophagy plays a protective role against TMV 24A + UPD induced cell death. Although autophagy seems to serve as an antiviral mechanism against TMV, it is still intriguing that there is no significant autophagic flux, as compared to TMV 24A + UPD. Autophagy has been shown to play an antiviral role [8], thus, it is possible that TMV subverts autophagy by an as yet unknown mechanism. A recent study has shown that Barley stripe mosaic virus (BSMV) interrupts autophagy by disrupting the interaction between ATG8 and ATG7 [47]. It is also plausible that the induction of autophagy flux by TMV 24A + UPD is a result of an altered host-pathogen interaction. Reactive oxygen species (ROS) can induce autophagy in mammalian and plant cells $[53,54]$. Autophagy plays a significant role to alleviate oxidative damage by reducing ROS $[53,54]$. Future studies will aim at identifying whether early infection by TMV 24A + UPD elicits an increased ROS which likely results in accelerated cell death.

\section{Conclusion}

Using the BioID technique, we have identified 67 proteins that interact with ATG8 in $N$. benthamiana plants infected with TMV and its more rapidly replicating mutant TMV 24A + UPD. A new ATG8 interacting protein, NbHYPK, was identified and verified for its interaction with ATG8. The interaction requires the presence of the UBA domain in NbHYPK. A recent study has described a new class of ATG8 adapters and receptors that exploit ubiquitininteracting motif sequences for high-affinity binding [55].

In summary, NbHYPK is involved in and may regulate autophagic processes by ATG8 during viral/TMV infection. Future studies will focus on elucidating the biological significance of NbHYPK chaperone activity and its relationship with other novel interacting partners identified in the functional proteome associated with ATG8 in N. benthamiana plants.

\section{Methods}

Plant materials, plasmid construction and agrobacterium infiltration

Nicotiana benthamiana seeds were obtained from Professor Dawei Li of China Agricultural University, Beijing, PRC. Seedlings and plants were grown in pots placed in growth rooms at $25^{\circ} \mathrm{C}$ under a 16 -h-light/8-h-dark cycle. NbATG8a (referred to as ATG8) was amplified using primers in Additional file 3: Table S2. The cDNA clone was used to construct vector PXY01 -35S-mCherryATG8a. The construct was transformed into Agrobacterium. For VIGS experiment, ATG5, ATG7 and ATG8 targets were amplified using primers listed in Additional file 3: Table S2. The resulting fragments were inserted into pTRV2. Together, pTRV1 and pTRV2 were introduced into Agrobacterium as previously described [56]. Downregulation of the gene expression for NbHYPK, ATG5, ATG7 and ATG8 was confirmed by qRT-PCR/RT-PCR (Fig. 6b; Additional file 1: Figure S5, S6b). Cloning was done using either ligation of restriction enzyme generated sticky ends (enzyme sites underlined) or Gibson Assembly ${ }^{\circ}$ cloning kit from NEB kit, see primers (prefix GA is used) in Additional file 3: Table S2.

E. coli biotin ligase BirA* was amplified from pcDNA 3.1 myc BioID plasmid using appropriate primers (Additional file 3: Table S2). Xba1 RE site and HA tag sequences were included in the forward primer while Sma1 was included in the reverse primer. The resulting fragment was digested with appropriate enzymes and inserted into vector PXY0 -35S-mCherry-ATG8. The resulting vector PXY01-mCherry-HABirA*-ATG8 (BirA*-ATG8) was confirmed by DNA sequencing. For the control plasmid, the fragment was inserted into PXY01-mCherry-X and resulted into PXY01-mCherry-HABirA* (BirA*). BioID clones were transformed into agrobacterium, and $2.5 \mathrm{mM}$ biotin incorporated into the agroinfiltration 
buffer. The clones were agroinfiltrated into fully expanded leaves and $2.5 \mu \mathrm{g}$ in vitro transcribed TMV 24A + UPD viral RNA was mechanically inoculated into the plants. The leaves were collected at $3 \mathrm{dpi}$ and total proteins extracted. Protein expression was verified by immunoblot using mCherry and ATG8 antibodies. For BiFC and subcellular localization, PCR fragments were amplified with primers shown in Additional file 3: Table S2 and cloned into respective vectors. $\mathrm{BiFC}$ vectors were transformed into Agrobacterium. The two cultures, nYFP-ATG8 and cYFP -NbHYPK, were mixed at a ratio of 1:1 and co-inoculated into $N$. benthamiana plants as previously described [28]. For subcellular co-localization, NbHYPK-GFP and RFPATG8 vectors were also co-cultured as above.

\section{Western blotting}

Total proteins were extracted from $N$. benthamiana leaves and separated by SDS-PAGE for immunoblot analysis with appropriate antibody. After proteins were transferred to nitrocellulose membrane, Ponceau $\mathrm{S}$ was used to stain proteins for loading control, according to manufacturer's protocol, before proceeding with blocking and antibody incubation. Protein gel band images were captured and converted into JPEG format and subjected to digital image analysis using ImageJ software version $1.52 \mathrm{a}[42]$.

\section{Confocal microscopy}

For autophagosome visualization, fully expanded leaves were agroinfiltrated with RFP-ATG8. After $24 \mathrm{~h}$, the infiltrated leaves were inoculated mechanically with $2.5 \mu \mathrm{g}$ in vitro transcribed RNA in GKP buffer ( $50 \mathrm{mM}$ glycine; 30 $\mathrm{mM} \mathrm{K}_{2} \mathrm{HPO}_{4}$, $\mathrm{pH}$ 9.2; $1 \%$ bentonite; $1 \%$ celite). After 3 days, leaves were syringe-infiltrated with $5 \mathrm{uM}$ of autophagy inhibitor E64d for $12 \mathrm{~h}$, immediately followed by confocal microscopy. For subcellular localization and BiFC, leaves were observed for fluorescence 3 days after agroinfiltration.

Live cell images were acquired from abaxial leaf epidermal cells using a Zeiss LSM510 microscope. GFP and RFP probes were excited at 488 and $561 \mathrm{~nm}$, respectively, and their fluorescent emissions detected at 495-550 and 570-610 nm, respectively. YFP was excited at $514 \mathrm{~nm}$ with an emission of $527 \mathrm{~nm}$. Confocal images were processed with ZEN (version 2011) and 578 Image J (version 1.48v) software.

\section{Tem}

Virus infected and/or autophagy inhibitor treated leaf samples were fixed in fixation medium (2\% paraformaldehyde $(\mathrm{w} / \mathrm{v}), 2.5 \%$ glutaraldehyde $(\mathrm{v} / \mathrm{v})$ in phosphate buffer (0.1 M, pH 7.0) overnight and post-fixed with $1 \%$ $\mathrm{OsO}_{4}$ in the phosphate buffer for $2 \mathrm{~h}$. After a series of ethanol dehydration, leaf samples were transferred to acetone for $20 \mathrm{~min}$ and then embedded in Spurr ${ }^{\circ}$ resin. The resin blocks were trimmed and ultrathin sectioned. After staining with $2 \%$ uranyl acetate and $1 \%$ alkaline lead citrate, the ultrathin sections were observed under a TEM (model JEOL JEM-1230).

\section{Yeast two hybrid $(\mathrm{Y} 2 \mathrm{H})$ assays}

The genes of interest (Additional file 3: Table S2) were cloned into either PGADT7 (AD) or PGBKT7 (BD) yeast plasmid. The recombinant vectors were transformed into AH109 yeast using lithium chloride as described by Matchmaker ${ }^{\circ}$ Yeast 2 Hybrid protocol. Transformed yeast colonies were grown in liquid media and diluted into 10-, 100- and 1,000-fold and spread over on SD/-Trp/-Leu, $\mathrm{SD} /$-His/-Trp/-Leu and SD/-His/-Trp/-Leu/-Ade plates. The plates were incubated at $30^{\circ} \mathrm{C}$ until colonies appeared 3 days later. Colonies that formed on more stringent selection using $\mathrm{SD} /-\mathrm{His} /-\mathrm{Trp} /-\mathrm{Leu}$ and $\mathrm{SD} /-\mathrm{His} /$ -Trp/-Leu/-Ade plates indicated physical interaction between the bait and prey proteins.

\section{BiolD sample preparation and MS/MS analysis}

The protein lysate was subjected to phenol extraction. The resulting pellet was dissolved in lysis buffer $(0.4 \%$ SDS, $500 \mathrm{mM}$ Nacl, $5 \mathrm{mM}$ EDTA, $1 \mathrm{mM}$ DTT, $50 \mathrm{mM}$ Tris-HCL, pH 7.4). Protein concentration was determined using Bradford assay. Streptavidin-coated Dynabeads $(200 \mu \mathrm{l})$ was gently added into $100 \mu \mathrm{g}$ of total proteins. The mixture was incubated at $4{ }^{\circ} \mathrm{C}$ overnight with gentle shaking. The beads containing bound biotinylated proteins were separated from unbound proteins using a magnetic stand. An aliquot of the unbound proteins was saved for Western blot.

The beads were washed several times (in $1 \mathrm{ml}$ of $1 \mathrm{X}$ PBS per wash with gently shaking, $5 \mathrm{~min}$ at room temperature), once in $2 \%$ SDS, once in $1 \%$ SDS, twice in $1 \%$ NP-40 and twice with 1 X PBS. The beads were left in the final wash. An aliquot of the beads was saved for Western blot analysis using streptavidin antibody (1: 2000). The beads were then subjected to MS analysis. Briefly, the beads were resuspended in $200 \mu \mathrm{l}$ triethylammonium bicarbonate.

(TEAB) (0.5 M, pH 8.5) with $4 \mu \mathrm{l}$ Tris(2-carboxyethyl)phosphine (TCEP) (100 mM stock) by gently mixing at $65^{\circ} \mathrm{C}$ for $1 \mathrm{~h}$. Four $\mu \mathrm{l}$ methyl methanethiosulfonate (MMTS) (200 mM stock) was added into the mixture above and incubated at room temperature for $15 \mathrm{~min}$. Tryspin was added into the beads at $12.5 \mathrm{ng} / \mu \mathrm{l}$ and incubated at $37^{\circ} \mathrm{C}$ for $16 \mathrm{~h}$ with gentle shaking. The beads were pelleted and the supernatant subjected to MS analysis. MS/MS data was searched against database of Nicotiana proteins using ProteinPilot ${ }^{\mathrm{Tx}}$. Data cleaning was carried out to remove insignificant hits. BirA*- 
ATG8 proteins that overlapped with the control BirA* proteins were also removed. Redundant and low quality proteins were excluded from the final analysis. The proteins were functionally classified. Gene ontology interactive network were summarized and plotted following published REVIGO protocol (http://revigo.irb.hr/ diagrams were generated using UGen Venn software (http://bioinformatics.psb.ugent.be/webtools/Venn/).

\section{Additional files}

Additional file 1: Figure S1. Full immunoblot images for Fig. 1a using ATG8 antibody. (a) Mock. (b) TMV. (c) TMV 24A + UPD. Figure S2. Expression of BirA* and BirA_ATG8 constructs. (a) Graphical representation of control BirA*and experimental plasmids

BirA*-ATG8. Full gel image for Fig. 2a carried out using anti-mCherry antiseri, for both (b) \& (c) and anti-ATG8 antiserum (* indicates a weak band). (d) to ascertain fusion protein expression. (e) Detection of RFP signals from agroinfiltrated N.benthamiana leaves to confirm

expression of RFP-fused BirA* and BirA*-ATG8. Figure S3. Full gel Immunoblot analysis of total biotinylated proteins for Fig. 2b and c. (a) Streptavidin-HRP blot of crude protein lysate. (b)Strep-HRP blot after DynabeadsTM purification. Abbreviations: $b$, beads; s, supernatant. Lane 1. WT- Biotin only. Lanes 2-3,-BirA*, lanes 4-5, BirA*-ATG8, lane 6-BirA*, lane 7, BirA*-ATG8, lane 8, BirA*, lane 9, BirA*-ATG8. Different treatments as follows: Samples $2-5$, biotin was infiltrated 3 days after agroinfiltration. Samples 6-9, biotin-infiltrated concurrently with agroinfiltration buffer. Samples 6 and 7, proteins extracted 3 dpi and samples 8 and

9 were collected 4 dpi. Treatments 6 and 7 were used for the final experiment. Figure S4. ATG8 directly interacts with NbHYPK but not NbHYPK $\triangle U B A$ and UBA-NbHYPK (Fig. 4). (a) GFP-NbHYPK, GFP-NbHYPK $\triangle \cup B A$, GFP-UBA-HYPK and RFP-ATG8 fusion proteins were detected in Western blot using GFP and RFP antibodies, respectively. (b) RFP-ATG8 aggregates with GFP-NbHYPK but not GFP -NbHYPK $\triangle U B A$ or GFP-UBA-NbHYPK. (c) Bimolecular fluorescence complementation (BiFC) analysis showed that ATG8 was able to associate with NbHYPK but ATG8-Yn did not associate with $x-Y c$ (plasmid without insert). NbHYPK-Yc also did not associate with $x-Y n$ (plasmid without insert). Figure S5. Analysis of gene down-regulation in ATG5/7/8-VIGS plants (Fig. 5). (a) Semi-quantitative RT-PCR expression analysis of ATG8 isoforms in ATG8-silenced and non-silenced Nicotiana benthamiana plants. (b) qRT-PCR analysis of ATG5 and ATG7 genes in control and VIGS-plants. Figure S6. TMV accumulation and NbHYPK relative expression in VIGS-NbHYPK plants (Fig. 6). (a) TMV coat

protein expression as detected by anti-TMV antibody. TMV coat protein is $\sim 17.5 \mathrm{kDa}$. Statistical significance was determined by Student's $t$ test ( $p=$.39). (b) Semi-quantitative qRT-PCR analysis of NbHYPK expression in VIGS silenced and non-silenced Nicotiana benthamiana plants. (ZIP 3798 kb)

Additional file 2: Table S1. Proteins identified through BiolD (DOCX $16 \mathrm{~kb}$ ) Additional file 3: Table S2. List of primers used in this study (DOCX $18 \mathrm{~kb}$ )

\section{Abbreviations}

ATG8: Autophagy 8; BiolD: Proximity dependent biotin identification; BirA*: Biotin protein ligase; G/Y/RFP: Green/Yellow/Red Fluorescent protein; HYPK: Huntingtin Interacting Protein K-like; MMTS: Methyl methanethiosulfonate; MS: Mass spectrometry; ROS: Reactive oxygen species; TCEP: Tris(2-carboxyethyl)phosphine; TEAB: Triethylammonium bicarbonate; TEM: Transmission electron microscope; UBA: Ubiquitin associated domain; VIGS: Virus induced gene silencing

\section{Acknowledgements}

The authors would like to thank Mr. Teck Kwang Lim at Protein and Proteomics Center, Department of Biological Sciences, NUS and Ms. Shaalini D/O Subramaniam and Mr. Ouyang Xuezhi at the TEM facility, Temasek Life Sciences Laboratory, Singapore, for their technical support.

\section{Authors' contribution}

MWM performed experiments, analysed and writing the manuscript. WZYT conducted the BiolD experiments and analysed the data. PPD assisted in designing experiments and optimization of protocols. NIN and SMW assisted in design of the study, discussing the results and editing the manuscript. All authors have read and approved the manuscript.

\section{Funding}

This work was financially supported by Singapore Ministry of Education through National University of Singapore research grant R-154-000-B23-114, Suzhou Bureau of Science and Technology grant SNG2018039, National Natural Science Foundation of China grant 31872639 and NUS-SINGA scholarship to Mercy W. Macharia. N. Naqvi acknowledges intramural funding from the Temasek Life Sciences Laboratory, Singapore. All funding played a role in the design of the study and collection, analysis, and interpretation of data and in writing the manuscript.

\section{Availability of data and materials}

All data generated or analysed during this study are included in this published article [and its supplementary information files].

Ethics approval and consent to participate

N/A

\section{Consent for publication}

N/A

\section{Competing interests}

The authors declare that they have no competing interests.

\section{Author details}

${ }^{1}$ Department of Biological Sciences, National University of Singapore, Singapore 119543, Singapore. ${ }^{2}$ Temasek Life Sciences Laboratory, Singapore 117604, Singapore. ${ }^{3}$ National University of Singapore Research Institute, Suzhou, Jiangsu 215123, People's Republic of China.

Received: 18 January 2019 Accepted: 9 July 2019

Published online: 19 July 2019

\section{References}

1. Nakashima A, Tanaka N, Tamai K, Kyuuma M, Ishikawa Y, Sato H, et al. Survival of parvovirus B19-infected cells by cellular autophagy. Virology. 2006:349(2):254-63.

2. Hofius D, Munch D, Bressendorff S, Mundy J, Petersen M. Role of autophagy in disease resistance and hypersensitive response-associated cell death. Cell Death Differ. 2011;18(8):1257-62.

3. Liu Y, Schiff M, Czymmek K, Talloczy Z, Levine B. Dinesh-Kumar S autophagy regulates programmed cell death during the plant innate immune response. Cell. 2005;121(4):567-77.

4. Agius C, Eamens AL, Millar AA, Watson JM, Wang MB. RNA silencing and antiviral defense in plants. Methods Mol Biol. 2012;894(1):17-38.

5. Haxim Y, Ismayil A, Jia Q, Wang Y, Zheng X, Chen T, et al. Autophagy functions as an antiviral mechanism against geminiviruses in plants. eLife. 2017:6(10):e23897.

6. Hafren A, Macia JL, Love AJ, Milner JJ, Drucker M, Hofius D. Selective autophagy limits Cauliflower mosaic virus infection by NBR1-mediated targeting of viral capsid protein and particles. Proc Natl Acad Sci U S A. 2017:114(10):E2026-e2035

7. Li F, Zhao N, Li Z, Xu X, Wang Y, Yang X, et al. A calmodulin-like protein suppresses RNA silencing and promotes geminivirus infection by degrading SGS3 via the autophagy pathway in Nicotiana benthamiana. PLoS Pathog. 2017;13(2):e1006213.

8. Hafrén A, Üstün S, Hochmuth A, Svenning S, Johansen T, Hofius D. Turnip mosaic virus counteracts selective autophagy of the viral silencing suppressor HCpro. Plant Physiol. 2017:176(1):649-62.

9. Li F, Zhang C, Li Y, Wu G, Hou X, Zhou X, et al. Beclin1 restricts RNA virus infection in plants through suppression and degradation of the viral polymerase. Nature Comm. 2018;9(1):1268.

10. Wang RY, Li K. Host factors in the replication of positive-strand RNA viruses. Chang Gung Med J. 2012;35(2):111-24. 
11. Lieberthal W. Macroautophagy: a mechanism for mediating cell death or for promoting cell survival? Kidney Int. 2008;74(5):555-7.

12. Fracchiolla D, Sawa-Makarska J, Martens S. Beyond Atg8 binding: the role of AIM/LIR motifs in autophagy. Autophagy. 2017;13(5):978-9.

13. Zeenko W, Ryabova LA, Spirin AS, Rothnie HM, Hess D, Browning KS, et al. Eukaryotic elongation factor $1 \mathrm{~A}$ interacts with the upstream pseudoknot domain in the $3^{\prime}$ untranslated region of Tobacco mosaic virus RNA. J Virol. 2002;76(11):5678-91.

14. Guo S, Kierzek E, Chen G, Zhou YJ, Wong SM. TMV mutants with poly(a) tracts of different lengths demonstrate structural variations in 3'UTR affecting viral RNAs accumulation and symptom expression. Sci Rep. 2015;5:18412.

15. Roux KJ, Kim DI, Raida M, Burke B. A promiscuous biotin ligase fusion protein identifies proximal and interacting proteins in mammalian cells. J Cell Biol. 2012;196(6):801.

16. Lin Q, Zhou Z, Luo W, Fang M, Li M, Li H. Screening of proximal and interacting proteins in Rice protoplasts by proximity-dependent Biotinylation. Front Plant Sci. 2017;8(10):749.

17. Khan M, Youn J-Y, Gingras A-C, Subramaniam R, Desveaux D. In planto proximity dependent biotin identification (BiolD). Sci Rep. 2018;8(1):9212.

18. Choudhury KR, Bucha S, Baksi S, Mukhopadhyay D. Bhattacharyya. Chaperone-like protein HYPK and its interacting partners augment autophagy. Eur J Cell Biol. 2016;95(6-7):182-94.

19. Pimkina J, Murphy ME. Murphy, interaction of the ARF tumor suppressor with cytosolic HSP70 contributes to its autophagy function. Cancer Biol Ther. 2011;12(6):503-9.

20. Guo B, Huang J, Wu W, Feng D, Wang $X$, Chen Y, et al. The nascent polypeptide-associated complex is essential for autophagic flux. Autophagy. 2014;10(10):1738-48.

21. Ramírez-Valle F, Braunstein S, Zavadil J, Formenti SC, Schneider RJ. elF4Gl links nutrient sensing by mTOR to cell proliferation and inhibition of autophagy. J Cell Biol. 2008;181(2):293.

22. Tumbarello DA, Waxse BJ, Arden SD, Bright NA, Kendrick-Jones J, Buss F. Autophagy receptors link myosin VI to autophagosomes to mediate Tom1dependent autophagosome maturation and fusion with the lysosome. Nat Cell Biol. 2012;14(10):1024-35.

23. Yamaguchi M, Noda NN, Nakatogawa H, Kumeta H, Ohsumi $Y$, Inagaki F. Autophagy-related protein 8 (Atg8) family interacting motif in Atg3 mediates the Atg3-Atg8 interaction and is crucial for the cytoplasmto-vacuole targeting pathway. J Biol Chem. 2010;285(38):29599-607.

24. Han S, Wang Y, Zheng X, Jia Q, Zhao J, Bai F, et al. cytoplastic Glyceraldehyde-3-Phosphate Dehydrogenases Interact with ATG3 to Negatively Regulate Autophagy and Immunity in Nicotiana benthamiana. Plant Cell. 2015;27(4):1316.

25. Gassen NC, Hartmann J, Schmidt MV, Rein T. FKBP5/FKBP51 enhances autophagy to synergize with antidepressant action. Autophagy. 2015;11(3):578-80.

26. Takeshige K, Baba M, Tsuboi S, Noda T, Ohsumi Y. Autophagy in yeast demonstrated with proteinase-deficient mutants and conditions for its induction. J Cell Biol. 1992;119(2):301-11.

27. Du A, Huang S, Zhao X, Feng K, Zhang S, Huang J, et al. Suppression of CHRN endocytosis by carbonic anhydrase CAR3 in the pathogenesis of myasthenia gravis. Autophagy. 2017;13(11):1981-94.

28. Xu G, Wang S, Han S, Xie K, Wang Y, Li J, et al. Plant Bax Inhibitor-1 interacts with ATG6 to regulate autophagy and programmed cell death. Autophagy. 2017;13(7):1161-75.

29. Xu M, Moresco JJ, Chang M, Mukim A, Smith D, Diedrich JK, et al. SHMT2 and the BRCC36/BRISC deubiquitinase regulate HIV-1 tat K63ubiquitylation and destruction by autophagy. PLoS Pathog. 2018;14(5): -e1007071.

30. Wang Z-H, Ren W-Y, Zhu L, Hu L-J. Plasminogen activator inhibitor-1 regulates LPS induced inflammation in rat macrophages through autophagy activation. Sci World J. 2014;2014(189168):1-12.

31. Ramesh N, Pandey UB. Autophagy dysregulation in ALS: when protein aggregates get out of hand. Front Mol Neurosci. 2017;10(33):263.

32. Ochaba J, Lukacsovich T, Csikos G, Zheng S, Margulis J, Salazar L, et al. Potential function for the huntingtin protein as a scaffold for selective autophagy. Proc Natl Acad Sci U S A. 2014;111(47):16889-94.

33. Xiao S, Gao W, Chen Q-F, Chan S-W, Zheng S-X, Ma J, et al. Overexpression of Arabidopsis acyl-CoA binding protein ACBP3 promotes starvation-induced and age-dependent leaf senescence. Plant Cell. 2010;22(5):1463-82.
34. Lin MH, Liu YC, Liu SY, Chen FC, Yang PJ, Li GH, et al. Clathrin-mediated endocytosis is required for ANE 30-100K-induced autophagy. J Oral Pathol Med. 2018;47(1):25-31.

35. Lin $\mathrm{P}$, Mobasher ME, Hakakian $\mathrm{Y}$, Kakarla $\mathrm{V}$, Naseem AF, Ziai $\mathrm{H}$, et al. Differential requirements for H/ACA ribonucleoprotein components in cell proliferation and response to DNA damage. Histochem Cell Biol. 2015: 144(6):543-58.

36. Lenk SE, Susan PP, Hickson I, Jasionowski T, Dunn WA Jr. Ubiquitinated aldolase B accumulates during starvation-induced lysosomal proteolysis. J Cell Physiol. 1999;178(1):17-27.

37. Ishida H, Yoshimoto K. Chloroplasts are partially mobilized to the vacuole by autophagy. Autophagy. 2008:4(7):961-2.

38. Izumi M, Wada S, Makino A, Ishida $\mathrm{H}$. The autophagic degradation of chloroplasts via rubisco-containing bodies is specifically linked to leaf carbon status but not nitrogen status in Arabidopsis. Plant Physiol. 2010; 154(3):1196-209.

39. Blommaart EF, Luiken JJ, Blommaart PJ, van Woerkom GM, Meijer AJ. Phosphorylation of ribosomal protein $\mathrm{S} 6$ is inhibitory for autophagy in isolated rat hepatocytes. J Biol Chem. 1995;270(5):2320-6.

40. Monastyrska I, He C, Geng J, Hoppe AD, Li Z, Klionsky DJ. Arp2 links autophagic machinery with the actin cytoskeleton. Mol Biol Cell. 2008;19(5):1962-75.

41. Kellner R, De la Concepcion JC, Maqbool A, Kamoun S, Dagdas YF. ATG8 expansion: a driver of selective autophagy diversification? Trends Plant Sci. 2017:22(3):204-14

42. Schneider CA, Rasband WS, Eliceiri KW. NIH image to ImageJ: 25 years of image analysis. Nat Methods. 2012;9(7):671.

43. Hofius D, Schultz-Larsen T, Joensen J, Tsitsigiannis DI, Petersen NH, Mattsson $\mathrm{O}$, et al. Autophagic components contribute to hypersensitive cell death in Arabidopsis. Cell. 2009;137(4):773-83.

44. Maeda Y, Oku M, Sakai Y. Autophagy-independent function of Atg8 in lipid droplet dynamics in yeast. J Biochem. 2017;161(4):339-48.

45. Ichimura $Y$, Kirisako T, Takao T, Satomi $Y$, Shimonishi $Y$, Ishihara N, et al. A ubiquitin-like system mediates protein lipidation. Nature. 2000; 408(6811):488-92.

46. Ketelaar T, Voss C, Dimmock SA, Thumm M, Hussey PJ. Arabidopsis homologues of the autophagy protein Atg8 are a novel family of microtubule binding proteins. FEBS Lett. 2004;567(2):302-6.

47. Yang M, Zhang Y, Xie X, Yue N, Li J, Wang XB, et al. Barley stripe mosaic virus gammab protein subverts autophagy to promote viral infection by disrupting the ATG7-ATG8 interaction. Plant Cell. 2018;30(7):1582-95.

48. Popelka $\mathrm{H}$, Klionsky DJ. Analysis of the native conformation of the LIR/ AlM motif in the Atg8/LC3/GABARAP-binding proteins. Autophagy. 2015;11(12):2153-9.

49. Zientara-Rytter K, Sirko A. Significant role of PB1 and UBA domains in multimerization of Joka2, a selective autophagy cargo receptor from tobacco. Front Plant Sci. 2014;5(10):13.

50. Arnesen T, Starheim KK, Van Damme P, Evjenth R, Dinh H, Betts MJ, et al. The chaperone-like protein HYPK acts together with NatA in cotranslational $\mathrm{N}$-terminal acetylation and prevention of huntingtin aggregation. Mol Cell Biol. 2010;30(8):1898-909.

51. N'Diaye EN, Kajihara KK, Hsieh I, Morisaki H, Debnath J, Brown EJ. PLIC proteins or ubiquilins regulate autophagy-dependent cell survival during nutrient starvation. EMBO Rep. 2009;10(2):173-9.

52. Choudhury KR, Raychaudhuri S, Bhattacharyya N. Identification of HYPKinteracting proteins reveals involvement of HYPK in regulating cell growth, cell cycle, unfolded protein response and cell death. PLoS One. 2012;7(12):e51415.

53. Chen YF, Liu H, Luo XJ, Zhao Z, Zou ZY, Li J, et al. The roles of reactive oxygen species (ROS) and autophagy in the survival and death of leukemia cells. Crit Rev Oncol Hematol. 2017;112:21-30.

54. Xiong Y, Contento AL, Bassham DC. Disruption of autophagy results in constitutive oxidative stress in Arabidopsis. Autophagy. 2007;3(3):257-8.

55. Marshall RS, Hua Z, Mali S, McLoughlin F, Vierstra RD. ATG8-binding UIM proteins define a new class of autophagy adaptors and receptors. Cell. 2019: In press. https://doi.org/10.1016/j.cell.2019.02.009.

56. Liu Y, Schiff M. Dinesh-Kumar S virus-induced gene silencing in tomato. Plant J. 2002;31(6):777-86.

\section{Publisher's Note}

Springer Nature remains neutral with regard to jurisdictional claims in published maps and institutional affiliations. 